\title{
Delayed Recovery from Anesthesia Following Suboccipital Craniotomy: A Case Report and Literature Review
}

\author{
Xiangyi Kong ${ }^{1,2}$, Haobo $\mathrm{Ma}^{3}$, Hao Deng ${ }^{2}$, Mark Young ${ }^{4}$ and Jingping Wang ${ }^{2^{*}}$ \\ ${ }^{1}$ Department of Neurosurgery, Peking Union Medical College Hospital, Chinese Academy of Medical Sciences, No. 1 Shuaifuyuan, Dongcheng District, Beijing 100730, \\ PR China \\ ${ }^{2}$ Department of Anesthesia, Critical Care and Pain Medicine, Massachusetts General Hospital, Harvard Medical School, Harvard University, 55 Fruit Street, Boston, \\ Massachusetts, 02114-3117, USA \\ ${ }^{3}$ Department of Anesthesia, Critical Care and Pain Medicine, Beth Israel Deaconess Medical Center, Harvard Medical School, 330 Brookline Ave, Boston, \\ Massachusetts, 02215, USA
}

${ }^{4}$ Department of Anesthesiology, Newton-Wellesley Hospital, 2014 Washington Street, Newton, Boston, Massachusetts, 02462, USA

"Corresponding author: Jingping Wang, Department of Anesthesia, Critical Care and Pain Medicine, Massachusetts General Hospital, Harvard Medical School, Harvard University, 55 Fruit Street, Boston, Massachusetts, 02114-3117, USA, Tel: 617-643-2729; Fax: 617-726-7536; E-mail: JWANG23@mgh.harvard.edu

Received Date: May 28, 2017; Accepted Date: June 26, 2017; Published Date: June 30, 2017

Copyright: (c) 2017 Kong X, et al. This is an open-access article distributed under the terms of the Creative Commons Attribution License, which permits unrestricted use, distribution, and reproduction in any medium, provided the original author and source are credited.

\begin{abstract}
Delayed awakening from anesthesia poses diagnostic challenges. The time for emergence from anesthesia is affected by patient factors, anesthetic factors, duration of surgery, and painful stimulation. However, delayed emergence can have unusual causes. We report a patient who, following termination of general anesthesia, did not regain consciousness for $6 \mathrm{~h}$ following surgery. Vital sign measurements, blood gas analysis, administrations of flumazenil and naloxone, and brain scan ruled out several possible causes for the delayed emergence. Apparently, the delayed emergence was a consequence of hypoperfusion of the brainstem during neurosurgical manipulations. To our knowledge, this is the first reported case of delayed anesthesia recovery on this basis.
\end{abstract}

Keywords: Delayed recovery from anesthesia; Suboccipital craniotomy; Brainstem hypoperfusion

\section{Highlights}

- Delayed emergence can have unusual causes and may present diagnostic dilemmas.

- Performance of early neurological assessment after anesthesia recovery is important in neurosurgery.

- Hypoperfusion of the brainstem due to neurosurgical manipulations is a possible cause for delayed emergence from general anesthesia.

\section{Introduction}

Delayed awakening from anesthesia is defined as failure to regain consciousness within 20-30 min after a surgical procedure [1]. The most common causes of delayed awakening are benign and mostly related to residual anesthetic effects. However, some causes are uncommon. Low incidence impedes the design of meaningful clinical trials investigating potential mechanisms and risk factors [2].

In neurosurgery, acute intracranial pathology is a feared possibility. To ensure that neurosurgical complications are diagnosed timely, it is desirable to perform an early neurological assessment immediately after anesthesia recovery [3,4]. Delayed anesthesia emergence precludes neurological assessment and may be caused by an array of benign or life-threatening conditions [5]. Patients who do not awaken promptly are urgently subjected to diagnostic procedures. Herein, we report a patient who failed to emerge from anesthesia after suboccipital craniotomy. He was normothermic, hemodynamically stable, and spontaneously breathing. The pupils were equal in size and reactive to light. A computed tomography (CT) brain scan and blood gas analysis were unremarkable. However, the patient could not follow commands. After $6 \mathrm{~h}$, recovery was improved and the trachea was extubated. It is likely that the failure to awaken was caused by hypoperfusion of the brainstem during the surgical manipulations.

\section{Case Presentation}

A 36-year-old man presented for elective left acoustic neuroma resection via suboccipital craniotomy. Symptoms had included intermittent progressive aggravated bilateral tinnitus, unstable gait, and balance disorder for 7 months. He complained of slight left hearing loss over the past 2 months. Six years previously, the patient had one episode of dysphagia, but it resolved without specific treatment. His past medical history included hypertension and hyperlipidemia. Oral medications were aspirin $325 \mathrm{mg}$ daily (stopped seven days prior to surgery), rosuvastatin $40 \mathrm{mg}$ daily, lisinopril $20 \mathrm{mg}$ daily, and propranolol $10 \mathrm{mg}$ twice a day. He had a history of mitral valve regurgitation and patent foramen ovale, with surgical repair in 2003. The preoperative transthoracic echocardiogram showed satisfactory reconstruction of mitral valves and normal estimated left ventricle ejection fraction. He was oriented and conscious. Visual fields and acuity were normal. The four limbs were neurologically normal, including the superficial and deep tendon reflexes. Ataxia and pathological signs were absent. He had no known drug allergies. Routine hematological test results were within normal limits. A preoperative brain CT scan showed a homogeneous mass at the left cerebellopontine angle, just below the left 7 th and 8 th nerve complex. The size was $4.3 \times 3.7 \times 3.1 \mathrm{~cm}$. The mass exhibited mild compression of the cerebellum, the adjacent brain stem (the pons and upper left medulla), the aqueduct of Sylvius, and the fourth ventricle. The CT 
images and clinical features were consistent with the diagnosis of acoustic neuroma.

The patient underwent left retromastoid suboccipital craniectomy and tumor resection. Following an intravenous induction with $2 \mathrm{mg}$ midazolam, $150 \mu \mathrm{g}$ fentanyl, $150 \mathrm{mg}$ propofol, and $100 \mathrm{mg}$ succinylcholine, the patient' $s$ trachea was intubated, and a radial arterial catheter was placed. He was positioned supine with his head turned to the right. Anesthesia was maintained with propofol infusion at $120-140 \mathrm{mcg} / \mathrm{kg} / \mathrm{min}$ and remifentanil infusion at $0.15-0.2 \mathrm{mcg} / \mathrm{kg} /$ $\mathrm{min}$. The patient also received $1 \mathrm{~g}$ vancomycin, $80 \mathrm{mg}$ gentamicin, 100 $\mathrm{mg}$ lidocaine, $10 \mathrm{mg}$ dexamethasone, $500 \mathrm{~mL} 20 \%$ mannitol, and 20 $\mathrm{mg}$ furosemide. The intradural micro-dissection was performed under the operating microscope, and the patient's hearing and facial nerve functions were monitored by an electrophysiological diagnostic system during the operation. While surgeons dissected the tumor capsule anterior to the left 7th and 8th nerve complex, the patient's hearing potentials declined and were not recovered afterwards. Throughout the operation, systolic blood pressure was maintained between 93 and 159 $\mathrm{mmHg}$, heart rate between 58 and 78 beats per minute, and oxyhemoglobin saturation between $99 \%$ and $100 \%$. The surgery lasted $8 \mathrm{~h}$. The majority of the tumor mass was removed during the last hour. The estimated blood loss was $100 \mathrm{~mL}$. The patient received 3,000 mL normal saline, and the total urine output was $3,925 \mathrm{~mL}$.

We reduced the propofol dose $30 \mathrm{~min}$ prior to the end of the surgery and stopped propofol and remifentanil infusions after the sterile dressing had been applied. During the next 30 minutes, the patient failed to recovery from anesthesia. His body temperature was 37 , blood pressure was $119 / 52 \mathrm{mmHg}$, heart rate was $67 / \mathrm{min}$, and spontaneous respiratory rate was $12 / \mathrm{min}$. The patient was initially unresponsive to painful stimuli. His pupils were equal in size and reactive to light, and the train-of-four responses were normal. In addition, an arterial blood gas sample was sent, revealing pH 7.37, PCO2 $41 \mathrm{mmHg}$, and PO2 322 $\mathrm{mmHg}$ during a $\mathrm{FiO}_{2}$ of 1.0. Glucose was $162 \mathrm{mg} / \mathrm{dL}$. Ionized calcium, sodium, and potassium were respectively $1.14,141$, and $4.2 \mathrm{mM}$. There was no EKG abnormality. An hour after the end of the surgery, upon firm painful stimulation, the patient would minimally move extremities and would not open eyes. Bispectral index (BIS) was used as measures of depth of anesthesia and sedation, and the BIS monitor value was in the $60 \mathrm{~s}$. The patient received $0.2 \mathrm{mg}$ flumazenil but did not improve. There was no arousal upon administration of $80 \mu \mathrm{g}$ naloxone. Three hours after the end of the surgery, there was no rejection of the endotracheal tube. A head CT scan was performed in order to rule out intracranial hematoma or brain-stem compression. There was no hydrocephalus, acute major vascular infarct, or intracranial hemorrhage. He was taken to the neurosurgical ICU. Patient was sedated with a small dose of propfol infusion only. At $6 \mathrm{~h}$ after the end of the surgery, the patient responded to voice commands, whereupon the trachea was extubated. He was oriented to person, place, and time, and there was grossly no neurological deficit.

\section{Discussion}

We report a patient who, following termination of general anesthesia, did not regain expected consciousness until $6 \mathrm{~h}$ after the end of the surgery. The delayed emergence was likely related to hypoperfusion of the brainstem during the surgical manipulations.

There is not a rigorous definition of delayed emergence from general anesthesia. With the uses of drugs such as desflurane and propofol, patients usually emerge in several minutes. According to
Zelcer et al., among 443 patients undergoing mixed surgeries, 9.5\% of the patients had impaired recoveries for 15-90 min postoperatively [6]. Delayed recovery from anesthesia usually refers to failure to regain consciousness within 20-30 min after the surgical procedure [1]. According to Misal et al., risk factors can be roughly divided into four types: patient factors, drug factors/pharmacological causes, duration and type of anesthetic used, and metabolic causes. Patient factors include extremes of age, male gender, underweight, comorbidities like preexisting cardiac and pulmonary diseases, structural disorders of central nervous system and psychological diseases, seizures, stroke, and insufficient postoperative pain. Drug factors include residual drug effects, potentiation of drugs by each other, drug interactions (such as that of meperidine with selective serotonin reuptake inhibitors or monoamine oxidase inhibitors), duration and type of anesthetic. Metabolic causes include hyper/hypoglycemia, electrolyte imbalance, hypothermia, and postsurgical respiratory failure caused by metabolic imbalance or primary muscle problems [7].

Residual anesthetics, analgesics and other risk factors listed above are the commonest reasons for delayed anesthesia recovery $[7,8]$. Nevertheless, unusual causes can also lead to delayed recovery. For example, in some patients with serotonin syndrome, mental status abnormality, autonomic hyper-reactivity, and neuromuscular abnormality may occur $[9,10]$. Rastogi et al. reviewed potential interactions between serotonergic drugs and opioids and their clinical implications [11]. Roy et al. reported a woman with venlafaxine (a serotonin norepinephrine reuptake inhibitor) use history, who presented with muscle rigidity which was aggravated by venlafaxine during the process of anesthesia emergence [12]. Central anticholinergic syndrome is also an uncommon reason for delayed emergence. Its symptoms vary from agitation to coma [13]. When it presents with depressed vigilance, it can be mistaken as residual anesthetic effects [2]. Neuro-excitatory symptoms such as seizures, opisthotonus, myoclonic movements, and twitching can also occur during the process of awaking from general anesthesia [14]. The causes for neuro-excitement include decreased inhibitory output from the formation reticularis or unbalanced cerebral inhibitory and excitatory pathways [14]. Other uncommon causes for delayed emergence include lidocaine infusion for arrhythmias, drug abuse, valproate toxicity, Hunter syndrome, thyroid failure, myxedema coma, dissociative coma, and hysteria [8]. Hysteria is diagnosed on the basis of exclusions of organic pathologies $[7,15]$.

In delayed recovery from anesthesia following neurosurgery, as a part of differential diagnostic algorithm, it is of utmost importance to consider conditions that can have a disastrous outcome if not timely detected. These include hemorrhage, ischemia, or brain swelling, which can be diagnosed through immediate brain $\mathrm{CT}$ or magnetic resonance imaging [4]. Residual anesthesia after neurosurgery may prevent timely diagnoses of intracranial pathologies such as cerebral infarction, herniation, and hematoma [3]. If patients are improperly positioned, especially in sitting operations, insufficiency of blood supply via the internal carotid artery system or vertebrobasilar arterial system may evoke hypoperfusion. Tracheal intubation and extubation, prolonged anticoagulant treatment and laryngoscopy may lead to hypertension, which is a noticeable cause for intracranial hemorrhage during anesthesia. For ventriculo-peritoneal shunt surgeries, the ventriculopuncture may result in small hemorrhages around the ventricular catheters (into subependymal areas or ventricles), and these may hinder the recovery of consciousness and present as delayed anesthesia emergence $[7,16]$. Munis et al. described a patient who failed to awaken from anesthesia and exhibited absence of brain-stem 
reflexes. They presumed that the patient's failure to awaken was caused by paralysis of brain-stem caused by migration of bupivacaine from the site of the injection [5].

In our patient, we used fentanyl for induction and remifentanil for anesthesia maintenance. Remifentanil's context-sensitive half-time is only $4 \mathrm{~min}$ so that its action is terminated shortly after stopping its infusion. Lingering effects of fentanyl may result in delayed awakening, especially in a patient with Hunter syndrome [17]. Opioids did not, however, explain the delayed awakening in our patient, because we did not see any improvement of his neurological status after the administration of naloxone. High sensitivity to benzodiazepines is also a consideration for the delayed emergence in our case. However, the specific antagonist flumazenil ruled out this possibility. We also ruled out atypical pseudocholinesterase by means of train-of-four tests. We also ruled out hypothermia or hypoglycemia. Three hours after the end of the surgery, a CT scan ruled out a brain lesion as a cause for delayed awakening. Therefore, our patient's delayed emergence was likely caused by hypoperfusion of brainstem during the surgical manipulations. Hypoperfusion is usually caused by low blood pressure, compression, or impaired venous drainage. In our case, arteries that supply the brainstem might have been compressed during the operation. On the other hand, patient was on propofol infusion for six hours. High dose propofol infusion for long duration will increase its context half time. It could be another cause of the delayed emergence. Additionally, patient received cold saline irrigation around brain stem during surgery, this cold saline might also cause delayed awakening.

\section{Conclusion}

We report a patient who, following termination of general anesthesia, did not regain consciousness and recovery completely until $6 \mathrm{~h}$ after the end of the surgery. He was initially unresponsive to painful stimuli. The delayed emergence was likely related to hypoperfusion of brainstem due to the surgical manipulations.

\section{Consent}

Written, informed consent was obtained from the patient for publication of this report.

\section{References}

1. Fuzaylov G, Kim AH, Rosow CE (2005) Delayed awakening from general anesthesia in a hypovolemic infant. Paediatr Anaesth 15: 435-436.
2. Tzabazis A, Miller C, Dobrow MF, Zheng K, Brock-Utne JG (2015) Delayed emergence after anesthesia. J Clin Anesth 27: 353-360.

3. Bruder N, Ravussin P (1999) Recovery from anesthesia and postoperative extubation of neurosurgical patients: a review. J Neurosurg Anesthesiol 11: $282-293$.

4. Morgenthaler K, Larsen B, Grundmann U, Silomon M (2005) Intracranial haemorrhage. A reason for delayed awakening after general anaesthesia. Anaesthesist 54: 450-454.

5. Munis JR, Marcukaitis AW, Sprung J (2006) Delayed emergence from anesthesia associated with absent brainstem reflexes following suboccipital craniotomy. Neurocrit Care 5: 206-209.

6. Zelcer J, Wells DG (1987) Anaesthetic-related recovery room complications. Anaesth Intensive Care 15: 168-174.

7. Misal US, Joshi SA, Shaikh MM (2016) Delayed recovery from anesthesia: A postgraduate educational review. Anesth Essays Res 10: 164-72.

8. Frost EA (2014) Differential diagnosis of delayed awakening from general anesthesia: a review. Middle East J Anaesthesiol 22: 537-548.

9. Nicolaou G, Lee D (2016) Methylene blue-induced serotonin syndrome presenting with ocular clonus and failure of emergence from general anesthesia. Can J Anaesth 63: 896-897.

10. Davis JJ, Buck NS, Swenson JD, Johnson KB, Greis PE (2013) Serotonin syndrome manifesting as patient movement during total intravenous anesthesia with propofol and remifentanil. J Clin Anesth 25: 52-54.

11. Rastogi R, Swarm RA, Patel TA (2011) Case scenario: opioid association with serotonin syndrome: implications to the practitioners. Anesthesiology 115: 1291-1298.

12. Roy S, Fortier LP (2003) Fentanyl-induced rigidity during emergence from general anesthesia potentiated by venlafexine. Can J Anaesth 50: 32-35.

13. Brown DV, Heller F, Barkin R (2004) Anticholinergic syndrome after anesthesia: a case report and review. Am J Ther 11: 144-153.

14. Islander G, Vinge E (2000) Severe neuroexcitatory symptoms after anaesthesia--with focus on propofol anaesthesia. Acta Anaesthesiol Scand 44: 144-149.

15. Maddock H, Carley S, McCluskey A (1999) An unusual case of hysterical postoperative coma. Anaesthesia 54: 717-718.

16. Deuri A, Goswami D, Samplay M, Das J (2010) Nonawakening following general anaesthesia after ventriculo-peritoneal shunt surgery: An acute presentation of intracerebral haemorrhage. Indian J Anaesth 54: 569-571.

17. Kreidstein A, Boorin MR, Crespi P, Lebowitz P, Barst S (1994) Delayed awakening from general anaesthesia in a patient with Hunter syndrome. Can J Anaesth 41: 423-426. 